Relations industrielles

Industrial Relations

\title{
L'aménagement du temps, par Jacques De Chalendar, Paris, Desclée de Brouwer, 1971, 120 pp.
}

\section{Laurent Bélanger}

Volume 29, numéro 2, 1974

URI : https://id.erudit.org/iderudit/028514ar

DOI : https://doi.org/10.7202/028514ar

Aller au sommaire du numéro

Éditeur(s)

Département des relations industrielles de l'Université Laval

ISSN

0034-379X (imprimé)

1703-8138 (numérique)

Découvrir la revue

Citer ce compte rendu

Bélanger, L. (1974). Compte rendu de [L'aménagement du temps, par Jacques De Chalendar, Paris, Desclée de Brouwer, 1971, 120 pp.] Relations industrielles / Industrial Relations, 29(2), 408-409. https://doi.org/10.7202/028514ar

Tous droits réservés @ C Département des relations industrielles de l'Université Laval, 1974
Ce document est protégé par la loi sur le droit d'auteur. L’utilisation des services d'Érudit (y compris la reproduction) est assujettie à sa politique d'utilisation que vous pouvez consulter en ligne.

https://apropos.erudit.org/fr/usagers/politique-dutilisation/ 
c'est le cas actuellement. La raison de ce refus à une demande syndicale qui aurait sans doute permis un meilleur équilibre des forces est liée à des considérations administratives: la nécessité de déterminer la liste des employés «désignés » avant qu'un bureau de conciliation ne soit nommé. Pourtant, cette justification ne tiendrait pas si une autre recommandation du rapport était adoptée, à savoir: la détermination par l'employeur des employés « désignés 》 six mois avant l'expiration d'une convention collective! De plus, plusieurs autres recommandations seront certainement fort mal accueillies par les syndicats: la possibilité pour l'employeur de recourir au lockout, la possibilité pour la CRT de « désigner » des employés additionnels si une grève se poursuit trop longtemps, l'extension de 7 à 14 jours de la période autour de laquelle le droit de grève est acquis après la remise du rapport de conciliation et l'interdiction de déclencher une grève si elle n'a pas été autorisée par l'agent négociateur. Il est quand même surprenant de constater ces restrictions additionnelles imposées à l'exercice du droit de grève devant le nombre relativement faible d'arrêts de travail survenus dans la Fonction publique fédérale au cours des dernières années. D'autant plus que ces nouvelles restrictions n'affecteront pas les syndicats qui ont déjà suffisamment de pouvoir de négociation mais plutôt ceux qui n'en ont déjà pas beaucoup. L'auteur du rapport, qui ne cache pas sa préférence pour l'arbitrage obligatoire au détriment du droit de grève, voudrait-il décourager d'éventuels agents négociateurs à choisir cette dernière procédure?

Sur un plan plus positif pour les syndicats, l'auteur suggère de permettre que les griefs de classification soient soumis à la procédure d'arbitrage en vertu des conventions collectives (alors qu'actuellement ils doivent être présentés individuellement à la Commission de la Fonction publique).

Enfin, Monsieur Finkelman propose de réorganiser la Commission des relations du travail dans la fonction publique de façon à en faire un organisme composé de membres qui y travailleraient à temps complet (plutôt que quelques jours par semaine). De plus, la Commission perdrait son caractère tripartite. Ses membres seraient nommés par le Gouverneur-général-en-conseil après consulta- tion avec les parties mais ils ne représenteraient plus officiellement ni les syndicats, ni les employeurs. La CRTFP serait composée d'un président, trois vice-présidents et au moins cinq nembres à plein temps ou plus selon la décision $\mathrm{du}$ Gouverneur-général-en-conseil. $\mathrm{La}$ CRTFP pourrait de plus s'adjoindre des conseillers à temps partiel sans droit de vote et représentant les parties impliquées.

Une recommandation intéressante est mise de l'avant qui consisterait à investir le CRTFP des pouvoirs de l'actuel Tribunal d'arbitrage de la Fonction publique. Le tribunal, on le sait, est chargé de désigner les arbitres dans les cas où les agents négociateurs ont choisi de recourir à l'arbitrage des différends. Si cette recommandation était acceptée, il n'y aurait plus nécessité de prévoir des mécanismes de révision des sentences arbitrales par la CRTFP.

Nous croyons que le réaménagement des fonctions de la Commission des relations de travail est la contribution la plus valable du rapport. Cependant, dans l'ensemble, celui-ci a été rédigé sous le signe de la préservation du «statu quo » et de la crainte de donner aux syndicats trop de pouvoirs. L'auteur et ses collaborateurs ont peut-être déjà pris pour acquis (consciemment ou non) que les relations patronales syndicales des années futures ne seraient pas aussi tranquilles qu'elle ne l'ont été jusqu'ici dans la Fonction publique du Canada

Université Laval

Jean BOIVIN

L'aménagement du temps, par Jacques De Chalendar, Paris, Desclée de Brouwer, $1971,120 \mathrm{pp}$.

C'est un ouvrage d'une lecture facile qui contient mille et une réflexions sur une phénomène que j'appellerais «l'entassement humain ». En effet, l'accroissement de la population à un rythme différent de celui de l'aménagernent de l'espace et du temps fait en sorte que nous nous retrouvons plusieurs au même endroit au même moment. Quelques-uns diront «on finit toujours par s'y habituer ». Mais, quand on y songe vraiment, on finit plutôt par trouver cela ennuyant et frustrant. Chalendar utilise une expression qui traduit bien le fait de se 
retrouver pour la plupart à poser les mêmes gestes au même moment: c'est le phénomène de l'encombrement. A titre d'exemples la bousculade pour attrapper le métro ou l'autobus, les embouteillages aux heures d'entrées et de sorties des bureaux, l'inaccessibilité aux terrains de camping durant le mois d'août, en France. Tous ces inconvénients dont la gravité s'accentue de jour en jour nous amènent à repenser l'utilisation du temps pour en effectuer de nouveaux découpages de même qu'à l'aménagement des espaces, puisque temps et espace vont de pair et que ce n'est pas uniquement par un aménagement du temps qu'on va régler le probème de la rareté relative de l'espace.

Dans les sociétés fortement industrialisées axées sur le «plus-être » collectif, on a appris à allouer et à découper le temps en fonction de critères économiques. "Time is Money 》 pour reprendre une expression de nos voisins, les Américains. Il faudrait arrêter de considérer le temps uniquement comme une ressource, une durée, une quantité. On découvrirait ainsi la valeur du moment, celui qui fait qu'une activité est plus intéressante parce qu'on a choisi le moment pour s'y adonner. Evidemment, je veux bien admettre que ce n'est pas possible pour tout le monde; cependant, ce nest pas une raison pour demeurer indifférent, puisque imaginer de nouvelles façons d'aménager son temps est déjà un début vers l'amélioration de la vie de travail et hors-travail.

C'est un peu le genre de réflexions que fait l'auteur et que je reprends dans mes propres termes. Mais, Jacques De Chalendar dépasse l'analyse du phénomène de l'encombrement pour élaborer des mécanismes qui permettraient de l'éliminer en partie. Il décrit des formes déjà connues et des formes récentes d'aménagement du temps; telles que le décalage des horaires fixes, les horaires personnalisés, les horaires flexibles. Il rappelle des expériences récentes telle que la semaine comprimée, l'étalement des congés au niveau de la semaine de travail.

Le phénomène des vacances annuelles retient aussi son attention. Il suggère d'en repenser la répartition par une division de l'année en deux parties, deux saisons: une période de pleine activité intense tant dans le secteur de l'écono- mie que celui de l'éducation entre les mois d'octobre et d'avril ; une période d'activité réduite de mai à septembre pendant laquelle une proportion de $25 \%$ à $30 \%$ de la population active serait en congé. A l'échelle de la vie de travail, il reprend des mesures déjà connues en particulier, l'abaissement de l'âge de la retraite obligatoire, la réglementation concernant les congés sabbatiques et l'entrée sur le marché du travail. L'ouvrage comprend aussi une annexe qui traite du budget-temps, du travail à temps partiel, de l'utilisation des temps libres. En conclusion, l'auteur fait un retour historique sur les notions de travail et de loisir pour se demander si la société de demain permettra un rapprochement de ces deux notions après les avoir pendant longtemps considérées comme opposées.

Sans chercher à effectuer une analyse systématique et approfondie des phénomènes qui retiennent son attention, Jacques De Chalendar réussit quand même à susciter le réflexion sur un sujet qui prend de plus en plus d'importance : celui de l'aménagement du temps. Cette réflexion doit se poursuivre puisqu'elle contient des possibilités d'amélioration du genre de vie qu'offre la société postindustrielle dans l'état actuel de son développement.

Université Laval

Laurent BELANGER

\section{A Practical Guide to Flexible Working} Hours, par Stephen Baum et W. McEwan Young, New Jersey, Noyes Data Corporation, 1974, 190 pp.

A ma connaissance, c'est un des premiers ouvrages publiés en anglais sur les horaires flexibles. Il couvre assez bien le sujet mais sous un angle très technique. L'auteur démarre par une réflexion sur la notion du travail à travers les âges et les nouvelles aspirations des travailleurs. Il situe les horaires flexibles parmi les nouvelles tentatives qui visent à redonner aux individus une certaine possibilité de choix dans l'organisation de leur travail, en particulier, l'aménagement des temps de travail. Les auteurs abordent ensuite les principes de base qui soustendent ce nouveau système d'horaire; les avantages et les inconvénients du point de vue de l'entreprise et de celui des diverses catégories de personnel. 